\title{
Exhaled breath analysis in lung transplant patients diagnosed with bronchiolitis obliterans syndrome
}

\author{
RM Hettiarachchi ${ }^{1,2,3}$, C Herbert, AR Glanville ${ }^{1,3}$ and PS Thomas ${ }^{1,2 *}$ \\ ${ }^{1}$ Inflammationand Infection Research Centre, Faculty of Medicine, University of NSW, NSW, Australia \\ ${ }^{2}$ Respiratory Medicine, Prince of Wales Hospital, NSW, Australia \\ ${ }^{3}$ Thoracic Medicine, St Vincent's Hospital, NSW, Australia
}

\begin{abstract}
Background: The efficacy of lung-transplantation is limited by chronic lung allograft dysfunction (CLAD). Currently CLAD is diagnosed via spirometry using the clinical surrogate of 'bronchiolitis obliterans syndrome'(BOS). Development of an alternative non-invasive diagnostic tool may be beneficial in decreasing the disease burden of CLAD in the lung transplant (LTx) population.
\end{abstract}

Aim: Determine the exhaled breath marker profile in LTx recipients as measured by potential biomarkers in the exhaled breath condensate (EBC).

Methods: $\mathrm{EBC}$ was collected with participants breathing tidally for 10 minutes. $\mathrm{pH}$ and concentrations of IL-8, IL-10, IL-13, IFN- $\gamma, \mathrm{NO}_{\mathrm{x}}$ and $\mathrm{H}_{2} \mathrm{O}_{2}$ were determined. LTx participants were followed for a year and progression in BOS stage was recorded.

Results: EBC was collected from 55 participants (10 non-BOS, 26 BOS and 19 controls). The LTx participants had higher levels of markers of non-specific inflammation compared to controls (median $\pm \mathrm{IQ}, \mathrm{H}_{2} \mathrm{O}_{2} 3.03 \pm 3.37 \mu \mathrm{M}$ vs. $1.60 \pm 2.06 \mu \mathrm{M}, \mathrm{p}<0.05, \mathrm{NO} 6.45 \pm 9.16 \mu \mathrm{M} v$ s. $0.63 \pm 0.68 \mu \mathrm{M}, \mathrm{p}<0.001$ ). $\mathrm{Of}$ the cytokines only IFN- $\gamma$ was consistently detectable in EBC samples but no significant difference was detected between the groups. IFN- $\gamma$ was higher in LTx participants with recent infection versus non-infected participants (mean \pm SD:64.31 $\pm 77.55 \mathrm{pg} / \mathrm{mL} v s .20 .45 \pm 32.41 \mathrm{pg} / \mathrm{mL} \mathrm{p}<0.05$ ). No marker was associated with BOS stage progression.

Conclusion: Markers of non-specific inflammation were elevated in LTx recipients, therefore exhaled breath analysis may provide a useful method for detecting inflammation in those with LTx and BOS.

\section{Introduction}

Exhaled breath condensate (EBC) analysis is developing as a novel and repeatable diagnostic tool in a number of respiratory conditions. It has the advantage of being able to be performed repeatedly within a short period of time, does not induce airway inflammation and may therefore be useful in monitoring disease progression or status in a number of respiratory conditions [1-3].

Biomarkers that have been detected in EBC include cytokines, markers of inflammation and markers of oxidative stress. Due to the potential for the use of EBC in clinical practice a joint American Thoracic Society/ European Respiratory Society (ATS/ERS) task force was developed to assess which biomarkers might be useful [2]. The task force concluded that none of the widely examined biomarkers have been sufficiently validated but opined that as collection and assaying techniques improve, EBC measurements might have the potential to assist clinical decisions [2].

Lung transplantation (LTx) is now an established therapy for selected recipients with advanced lung disease, but long-term survival is limited primarily by the development of chronic lung allograft dysfunction (CLAD) [4]. The most common cause of CLAD is obliterative bronchiolitis, which is diagnosed most reliably by open lung biopsy, which is invasive, and impractical for repeated surveillance as a routine. Physiological (e.g. lung function) and radiological studies are limited by a lack of sensitivity and specificity, and as a compromise, many LTx centers recommend surveillance bronchoscopy with transbronchial lung biopsies and bronchoalveolar lavage post-transplant to screen for allograft rejection, infection and other changes in airway lining fluid [5-8]. Nevertheless, it is clear that surveillance studies as currently performed are not universally successful in identifying the development of CLAD and the associated morbidity and mortality [8].

There is therefore a need for new tools to monitor those with LTx and to detect early CLAD. EBC biomarker analysis has the potential as a non-invasive tool to assess the onset and monitor the progress of conditions such as CLAD, allograft infection and acute allograft rejection after LTx but there are, as yet, no supportive data demonstrating clinical utility in the literature. Some studies have suggested that detection of volatile organic compounds in the breath may have a good negative predictive value for the absence of rejection, and be able to detect cardiac transplant rejection [9]. Prospective studies using this type of monitoring are awaited.

Correspondence to: Professor Paul S. Thomas, Consultant Physician, Department of Respiratory Medicine, Prince of Wales Hospital, Randwick, NSW 2031, Australia, Tel: +61293824620; Fax: +61293824627; E-mail: paul.thomas@ unsw.edu.au

Received: February 22; Accepted: March 26, 2017; Published: March 29, 2017 


\section{Methods}

\section{Patient recruitment}

This study was approved by the Prince of Wales and St. Vincent's Hospitals' Human Research Ethics Committees. Successive LTx recipients attending St. Vincent's Hospital Heart-Lung Clinic were invited to take part in the study and written, informed consent was obtained prior to samples being obtained. Single LTx recipients were excluded, as were those with a repeat LTx. BOS was diagnosed as per ISHLT guidelines and included those in stage BOS-0p [5,10].

Control participants were recruited from volunteers at the Prince of Wales and St. Vincent's Hospitals. Control participants were defined as individuals with no significant history of systemic or pulmonary diseases and had not smoked in the previous two years and matched for age and gender.

\section{Sample collection}

Using a previously validated collection technique, participants breathed tidally through a unidirectional valve connected to an inverted 'J-shaped' glass tube condenser including a saliva trap [11,12]. The glass collection chamber was surrounded by wet ice contained in an insulated flask. Collection continued until sufficient EBC was collected or the subject was unwilling to continue.

EBC samples were de-aerated by $400 \mathrm{~mL} / \mathrm{min}$ argon gas for one minute over the surface of the sample to remove carbon dioxide [13]. Aliquots of $120 \mu \mathrm{L}$ of sample were stored in polypropylene tubes at $-80^{\circ} \mathrm{C}$ until analysis.

\section{Biomarker measurement}

Hydrogen peroxide: Reagents were purchased from Sigma-Aldrich (Sydney, Australia) unless otherwise indicated. Hydrogen peroxide $\left(\mathrm{H}_{2} \mathrm{O}_{2}\right)$ was analysed using a colorimetric assay using the horseradish peroxidase-catalysed oxidation of tetramethylbenzidine (TMB) with limit of detection (LOD) of $0.22 \mu \mathrm{M}$ [14]. A $100 \mu \mathrm{L}$ aliquot of EBC was added to $10 \mu \mathrm{L}$ of $420 \mu \mathrm{M}$ TMB in $0.42 \mathrm{M}$ potassium citrate buffer and $10 \mu \mathrm{L} 52.5 \mathrm{U} / \mathrm{mL}$ horseradish peroxidase before being incubated for 20 minutes at room temperature. The reaction was stopped by adding $15 \mu$ Lof $2 \mathrm{M}$ sulfuric acid $\left(\mathrm{H}_{2} \mathrm{SO}_{4}\right)$ to produce 3,3'5,5'-tetramethyl1,1'-diphenoquinone-4,4'-diamine. The absorbance was read using a SpectraMax M2 plate reader (SpectraMaxPlus Plate Reader, Molecular Devices, Surrey, UK) at $450 \mathrm{~nm}$. Concentrations of $\mathrm{H}_{2} \mathrm{O}_{2}$ were interpolated from the standard curve.

Nitrogen oxides: Total nitrogen oxides $\left(\mathrm{NO}_{\mathrm{x}}\right)$ was analysed using a fluorometric assay based on the Greiss reaction with a LOD of $1.25 \mu \mathrm{M}$ [15]. Nicotinamide adenine dinucleotide phosphate (NADPH, $20 \mu \mathrm{L}$ of $0.25 \mathrm{mM}), 10 \mu \mathrm{L}$ of $50 \mu \mathrm{M}$ flavine adenine dinucleotide (FAD) and $20 \mu \mathrm{L}$ of $250 \mathrm{IU} / \mathrm{L}$ of nitrate reductase were added to $50 \mu \mathrm{L}$ of sample and incubated for 1 hour at $37^{\circ} \mathrm{C}$. 2,3-diaminonaphthalene (DAN, 10 $\mu \mathrm{L}$ ) was then added and incubated for 10 minutes in the dark to react with nitrite to produce $1(\mathrm{H})$-napthotriazole. The reaction was stopped by adding $10 \mu \mathrm{L}$ of $2.8 \mathrm{M}$ sodium hydroxide and fluorescence was read using a SpectraMax M2 plate reader, excitation $360 \mathrm{~nm}$, emission 395 $\mathrm{nm}$. Concentrations of $\mathrm{NO}_{\mathrm{x}}$ were interpolated from the standard curve.

Cytokines: Cytokine analysis for IL-8, IL-10, IL-13 and IFN- $\gamma$ was performed using a custom Bio-Plex cytokine assay (Bio-Rad Laboratories, Hercules, USA) as per the manufacturer's instructions. The LODs of the cytokines were $0.5 \mathrm{pg} / \mathrm{mL}, 0.9 \mathrm{pg} / \mathrm{mL}, 2.1 \mathrm{pg} / \mathrm{mL}$ and $19.3 \mathrm{pg} / \mathrm{mL}$ respectively. The filter plate was pre-wet using $100 \mu \mathrm{L}$ of assay buffer. An aliquot of $50 \mu \mathrm{L}$ of antibody coupled magnetic beads was added to each well before being washed 2 times with wash buffer. Sample $(50 \mu \mathrm{L})$ was added before incubating in the dark for 30 minutes at room temperature and shaking at 300 RPM. Wells were then washed 3 times with wash buffer, $25 \mu \mathrm{L}$ of detection antibody was added and the plate was once again incubated in the dark for 30 minutes at room temperature and shaking at 300 RPM. Wells were again washed 3 times with wash buffer, $50 \mu \mathrm{L}$ of streptavidin-phycoerythrin was added and the plate was once again incubated in the dark for 10 minutes at room temperature and shaking at 300 RPM. Wells were again washed 3 times with wash buffer before $125 \mu \mathrm{L}$ of assay buffer was added to re-suspend the beads before shaking at 1100 RPM for 30 seconds. The plate was read using the Luminex 100xMAPTM system (Luminex, Austin, USA) and data analysis was performed with Bio-Plex Manager software version 6.0 (Bio-Rad Laboratories). Concentrations of the cytokines were interpolated from standard curves. Only results with more than $50 \%$ of the samples above the limit of detection were used in analysis.

pH: $\mathrm{pH}$ was measured using a $\mathrm{pH}$ probe (ISFET, Hach Co., Loveland, USA). The $\mathrm{pH}$ meter was calibrated daily using a 3-point calibration with a measurement range 2-12 $( \pm 0.1 \mathrm{pH})$.

\section{Follow up}

All LTx participants were followed for approximately one year after samples were obtained to monitor progression of BOS stage. Participants who were BOS stage 3 and hence could not progress in stage were omitted from follow up analysis.

\section{Statistical analysis}

Data were analysed using SPSS software Version 21 (IBM Co., New York, USA). Parametric data were analysed using analysis of variance (ANOVA) and Dunnett's multiple comparison post-hoc test for comparisons between groups. Non-parametric data were analysed using Kruskal-Wallis test and Dunn's post-hoc test. Parametric data are expressed as mean \pm [SEM] and non-parametric data as median \pm [IQR]. $p$ values less than 0.05 were considered significant.

\section{Results}

Participants were recruited (total $=55$ ) comprising $10 \mathrm{LTx}$ recipients without BOS, 26 LTx recipients with BOS and 19 healthy controls. Demographic data are summarised in Table 1. All LTx recipients were taking triple-drug maintenance immunosuppressive therapy which includes a calcineurin inhibitor, corticosteroid and antiproliferative agent. There was insufficient EBC to run all assays on all samples. Parametric data is presented as mean \pm standard deviation (M $\pm \mathrm{SD})$ and non-parametric data is presented as median \pm inter quartile range $(\mathrm{M} \pm \mathrm{IQ})$

\section{Hydrogen Peroxide}

EBC samples from 9 LTx recipients without BOS, 23LTx recipients with BOS and 18 healthy control participants were used in this assay. $\mathrm{H}_{2} \mathrm{O}_{2}$ was detectable in all but two samples and in these, an arbitrary value of half the detectable limit of the assay was assigned. Taking the overall LTx group there was a significantly higher median $\mathrm{H}_{2} \mathrm{O}_{2}$ in the EBC of LTx recipients compared to the healthy controls (median \pm IQ: $3.03 \pm 3.37 \mu \mathrm{M}$ vs. $1.60 \pm 2.06 \mu \mathrm{M}, \mathrm{p}<0.05$ ), but there were no significant differences between the BOS, non-BOS and control groups (median \pm IQ: $2.94 \pm 5.00 \mu \mathrm{M} v s .3 .12 \pm 2.10 \mu \mathrm{M} v s .1 .60 \pm 2.06 \mu \mathrm{M}$ ). $\mathrm{H}_{2} \mathrm{O}_{2}$ was not correlated to a recent history of infection, acute rejection or previous history of antibody-mediated rejection. 
Table 1. Patient characteristics.

\begin{tabular}{|c|c|c|c|c|}
\hline & $\begin{array}{c}\text { non-BOS } \\
(\mathbf{n}=\mathbf{1 0})\end{array}$ & $\begin{array}{c}\text { BOS } \\
(\mathbf{n = 2 6})\end{array}$ & $\begin{array}{c}\text { Controls } \\
(\mathbf{n = 1 9 )}\end{array}$ & $\begin{array}{c}\text { Transplant } \\
(\mathbf{n}=\mathbf{3 6})\end{array}$ \\
\hline Male: Female & $7: 3$ & $10: 16$ & $10: 9$ & $17: 19$ \\
\hline Age (years); mean \pm SD & $45.1 \pm 13.8$ & $43.0 \pm 16.4$ & $42.3 \pm 14.3$ & $44.6 \pm 15.3$ \\
\hline Transplant indication (CF:non-CF) & $5: 5$ & $8: 18$ & N/A & $13:$ \\
\hline Transplant type (BSSLTx:HLTx) & $10: 0$ & $23: 3$ & N/A & $33: 3$ \\
\hline BOS Stage (0p:I:II:III) & N/A & $3: 6: 8: 9$ & N/A & $3: 6: 8: 9$ \\
\hline Medications & & & & \\
Calcineurin inhibitor & 10 & 26 & 0 & 36 \\
Cell-cycle inhibitor & 10 & 26 & 0 & 36 \\
Systemic corticosteroids & 10 & 26 & 0 & 36 \\
Proton pump inhibitor & 10 & 25 & 1 & 35 \\
\hline Time post-transplant (days) & $2108 \pm 2122$ & $2694 \pm 1559$ & N/A & $2531 \pm 1722$ \\
\hline Infections (number affected) & & & & \\
Bacterial & 3 & 5 & N/A & 8 \\
Viral & 4 & 8 & N/A & 12 \\
\hline Rejection (number affected) & & & & \\
Acute & 4 & 4 & N/A & 8 \\
Antibody mediated & 1 & 4 & N/A & 5 \\
\hline Lung Function & & & & \\
FEV1 baseline (L/s) & $3.15 \pm 0.72$ & $2.86 \pm 0.93$ & N/A & $2.94 \pm 0.87$ \\
FEV1 (L/s) & $2.67 \pm 0.55$ & $1.48 \pm 0.76$ & N/A & $1.81 \pm 0.88$ \\
FEV1 predicted (\%) & $82 \pm 17$ & $51 \pm 25$ & N/A & $60 \pm 26$ \\
\hline
\end{tabular}

Data are presented as mean \pm standard deviation.

BOS=Bronchiolitis Obliterans Syndrome; CF=Cystic Fibrosis; BSSLTx=Bilateral Sequential Single Lung Transplant; HLTx=Heart Lung Transplant; FEV1=Forced Expiratory volume in 1 second

\section{Nitrogen Oxides}

EBC samples from 10LTx recipients without BOS, 18LTx recipients with $\mathrm{BOS}$ and 15 controls were available for the assay. $\mathrm{EBC} \mathrm{NO}$ was detectable in 8/10LTx recipients without BOS, 15/18 LTx recipients with BOS and 4/15 control subjects. A value of half the detectable limit was assigned to samples where the level of $\mathrm{NO}_{\mathrm{x}}$ was undetectable. Overall, LTx recipients had a significantly higher level of $\mathrm{EBC} \mathrm{NO}_{\mathrm{x}}$ compared to the healthy controls (median \pm IQ: $6.45 \pm 9.16 \mu \mathrm{M} v s .0 .63 \pm 0.68 \mu \mathrm{M}$, $\mathrm{p}<0.001$ ). Likewise, median levels of $\mathrm{EBC} \mathrm{NO}_{\mathrm{x}}$ in LTx recipients with or without BOS were both significantly elevated compared to the control group (median \pm IQ: $5.62 \pm 12.24 \mu \mathrm{M} v$ s. $0.63 \pm 0.68 \mu \mathrm{M}, \mathrm{p}<0.005,7.72$ $\pm 9.22 \mu \mathrm{M} v s .0 .63 \pm 0.68 \mu \mathrm{M}, \mathrm{p}<0.05)$, however there was no significant difference between LTx recipients with and without BOS. $\mathrm{NO}_{\mathrm{x}}$ was not correlated with infection or rejection (Figures 1 and 2).

\section{Cytokines}

Samples from LTx recipients without BOS $(\mathrm{n}=9)$, LTx recipients with BOS $(n=26)$ and controls $(n=19)$ were available for the assay. From these 54 samples, IL-8 was detected in 6, IL-10 in 7, IL-13 in none, but IFN- $\gamma$ was detected in 46 samples. Hence, only IFN- $\gamma$ data were analysed. A value of half the limit of detection was assigned to samples where the level of IFN- $\gamma$ was undetectable. There were no significant differences between the BOS, non-BOS and control groups (mean \pm SD: $41.34 \pm 65.26 \mathrm{pg} / \mathrm{mL}$ vs. $20.45 \pm 73.80 \mathrm{pg} / \mathrm{mL}$ vs. $37.50 \pm 42.31$ $\mathrm{pg} / \mathrm{mL}$ ). Likewise, the overall LTx group did not differ significantly from the healthy controls (mean \pm SD: $37.50 \pm 67.85 \mathrm{pg} / \mathrm{mL} v s .37 .50$ $\pm 42.31 \mathrm{pg} / \mathrm{mL}$ ). IFN- $\gamma$ was significantly higher in LTx participants with a recent infection (mean $\pm \mathrm{SD}$, infection: $64.31 \pm 77.55 \mathrm{pg} / \mathrm{mL} v s$. non-infected $20.45 \pm 32.41 \mathrm{pg} / \mathrm{mL} \mathrm{p}<0.05$ ). A recent history of acute rejection or previous antibody-mediated rejection were not correlated with EBC IFN- $\gamma$ levels.
pH

EBC $\mathrm{pH}$ was measured in all 55 participants. There were no significant differences between the BOS, non-BOS and control groups (mean \pm SD: $6.8 \pm 1.4$ vs. $7.5 \pm 1.6 v s .6 .6 \pm 1.0$ respectively). When the data from the participants with and without BOS were amalgamated into a single LTx group there was again no significant difference between the LT participants and controls $(\mathrm{M} \pm \mathrm{SD}$ : $7.0 \pm 1.2$ vs. 6.6 \pm 1.0 ] but $\mathrm{pH}$ was significantly higher in LTx participants with a history of acute rejection $(\mathrm{M} \pm \mathrm{SD}: 7.6 \pm 0.7$ vs. $6.7 \pm 2.1)$. $\mathrm{pH}$ was not correlated with a recent history of infection or previous history of antibody-mediated rejection.

\section{Follow up and progression of BOS}

Stage 3 BOS participants $(n=9)$ were omitted from follow up analysis as they could not demonstrate further progression. Of the remaining LTx participants, 9 progressed in stage. Three of these

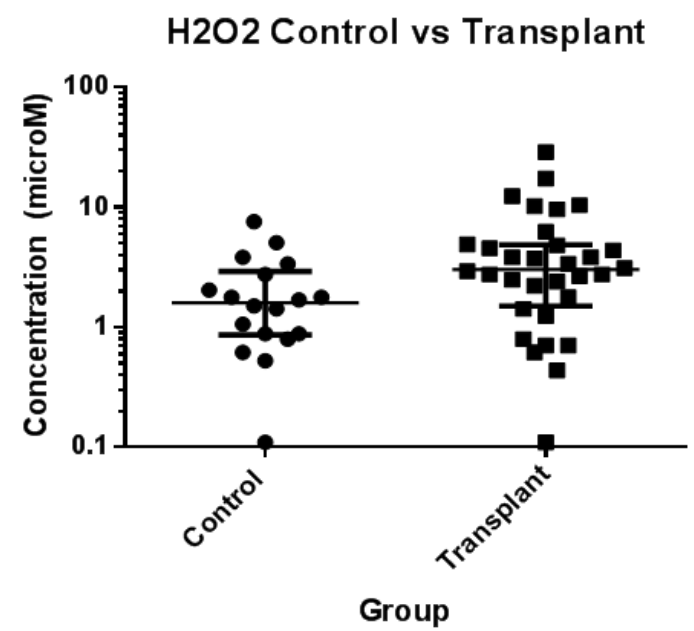

EBC=Exhaled Breath Condensate; $\mathrm{H}_{2} \mathrm{O}_{2}=$ Hydrogen Peroxide; LTx=Lung Transplant Figure 1. EBC Hydrogen peroxide concentrations $(\mu \mathrm{M})$ were significantly higher in the LTx group compared to controls $(\mathrm{p}<0.05)$.

\section{NOx Control vs Transplant}

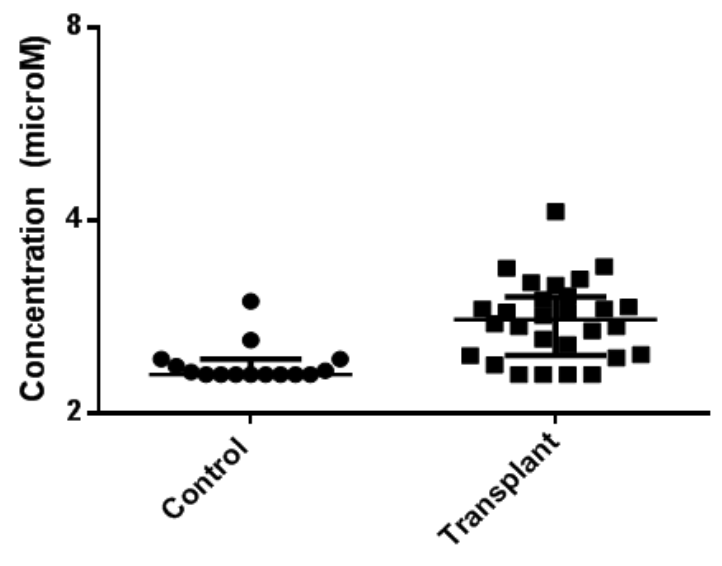

Group

EBC $=$ Exhaled Breath Condensate; NOx=Nitrogen Oxides; LTx=Lung Transplant Figure 2. EBC nitrogen oxide concentration $(\mu \mathrm{M})$ were significantly higher in the LTx group compared to controls $(\mathrm{p}<0.001)$. 
progressed from Stage 0 to 0 -p, two from Stage 0 -p to 1, one from Stage 1 to 2 and three from Stage 2 to 3 . There was no significant correlation between stage progression and any of the measured biochemical EBC variables of $\mathrm{H}_{2} \mathrm{O}_{2}, \mathrm{NO}_{\mathrm{x}}$, IFN- $\gamma$ or $\mathrm{pH}$.

\section{Discussion}

This is the first study to examine EBC biomarkers in lung transplantation with progression to chronic rejection. Although other studies have examined EBC $\mathrm{pH}$ and cytokine levels, this study is the first to report on the non-specific markers of inflammation and oxidative stress, nitrogen oxides and hydrogen peroxide. It has shown that, despite immunosuppression, these non-specific markers of inflammation are elevated in the EBC of patients with lung transplants when compared to controls. There was no difference in the levels of these biomarkers between the participants with BOS compared to those without, but longer term follow-up EBC might have been helpful. All other markers of inflammation including cytokines and $\mathrm{pH}$ did not differ between those with lung transplants and controls. The biomarker levels did not correlate with progression of BOS at one year follow up in participants with lung transplants, although the numbers were small.

The finding that lung transplant participants had higher levels of markers of oxidative stress and inflammation in the EBC of lung transplant recipients, despite immunosuppression, is consistent with other studies [16-19]. No significant differences between the BOS and non-BOS groups were able to be demonstrated by the breath analysis. The heterogeneous nature of both the indication for transplantation, as well as the complications and immunosuppression means that the subgroups in this study are limited by small sample sizes and between subject variability, but the results do allow estimates for suitable power in future studies. We have used the consensus diagnostic criteria for BOS, but a much larger study could examine subtypes of BOS, any markers suggesting progression of BOSO-p and any progression of disease [20].

The cytokines IL-8, IL-10 and IL-13 were undetectable in the majority of the samples, and the levels detected did not differ between the groups. Of the cytokines measured, only IFN- $\gamma$ was detected in the majority of the samples. These results are in contrast to an earlier study which described a more than three-fold increase of IFN- $\gamma$ in the EBC of participants with BOS compared to those without [19]. This study however used a different EBC collection apparatus and concentrated their samples using the technique of lyophilisation. In addition to this they pooled samples from participants from each group and reported relative increases in levels. Our study suggested that recent infection correlated with increased EBC levels of IFN- $\gamma$ which is consistent with its role as a pro-inflammatory Th1 cytokine. This would mean that its role in differentiating between those with and without BOS would be limited due to lack of specificity.

The low levels of cytokines in EBC are in part due to the sample representing highly diluted airway lining fluid, with even when using highly sensitive available commercial assays. The cytokines studied have previously demonstrated a significant increases in the EBC of those with BOS and were chosen for this study after considering the current theoretical pathophysiology of this disorder [16,19]. Due to this difficulty in detecting cytokines in EBC, any clinical utility of cytokine examination will need refinement in the future with more sensitive assays. Lyophilisation and subsequent reconstitution in a smaller volume is a technique which can be used on EBC samples and can concentrate biomarkers by a factor up to thirty-times [21]. This or other concentrating techniques may help in the detection and subsequent analysis of cytokines in EBC and should be used in future studies if cytokine levels are to be reliably detected. Comparison with BAL may be appropriate but few studies have shown good correlation between the two techniques and they probably sample different lung compartments [17]. Those with infection were not recruited due to the complexity of adding a further variable in the analyses.

There were several methodological limitations of in this study. Some subjects were mildly breathless, therefore only small amounts of EBC able to be collected and hence not all the assays were able to be performed for each participant. EBC collection was unsuccessful in a number of the potential subjects, mainly as they felt breathless during the procedure. Although the EBC collection only requires tidal breathing, some found this procedure provoked a sensation of breathlessness.

Another limitation was the amount of EBC that was able to be obtained from the participants using wet ice as a coolant in the collection apparatus, at approximately $4^{\circ} \mathrm{C}$ as it has been shown that the yield of $\mathrm{EBC}$ and associated biomarkers is increased using lower condensing temperatures [11,22]. The use of an alternative EBC collection apparatus would also be useful to validate the differences found in the non-specific markers of $\mathrm{pH}, \mathrm{NO}_{\mathrm{x}}$ and $\mathrm{H}_{2} \mathrm{O}_{2}$. Although every effort was made to limit external influences that may influence the study by conforming to published guidelines and controlling for confounding factors such as recent meals/beverages, smoking history, mouthwash, etc, it has been shown that the method of collection of EBC may influence the biomarker profile found, in particular with these non-specific markers. It has been suggested that $\mathrm{NO}_{\mathrm{x}}$ is a biomarker that is especially subject to contamination from collection devices and the surrounding environment and hence the results from our study do require validation by other studies $[11,17,23]$.

In conclusion EBC can be used to demonstrate inflammation noninvasively in the lungs of those with lung transplants. Markers of nonspecific inflammation such as nitrogen oxides and hydrogen peroxide are inexpensive, simple assays of markers that are reliably detected in EBC compared to cytokine levels, and the clinical applicability of these markers warrants further research [14,24]. Further development is needed to assess the potential of cytokines in the EBC as a diagnostic tool with more sensitive assays or methods of concentrating EBC to ensure that the appropriate analyses may be performed in all subjects.

\section{References}

1. Montuschi P (2007) Analysis of exhaled breath condensate in respiratory medicine: methodological aspects and potential clinical applications. Ther Adv Respir Dis. 1: 5-23. [Crossref]

2. Horváth I, Hunt J, Barnes PJ, Alving K, Antczak A, et al. (2005) Exhaled breath condensate: methodological recommendations and unresolved questions. Eur Respir J. 26: 523-548. [Crossref]

3. Thomas PS, Lowe AJ, Samarasinghe P, Lodge CL, Huang Y, et al. (2013) Exhaled breath condensate in paediatric asthma: promising new advance or pouring cold water on a lot of hot air? A systematic review. Ped Pulmonol. 48: 419-442. [Crossref]

4. Finlen Copeland CA, Snyder LD, Zaas DW, Turbyfill WJ, Davis WA, et al. (2010) Survival after bronchiolitis obliterans syndrome among bilateral lung transplant recipients. Am J Respir Critl Care Med. 182: 784-789. [Crossref]

5. Estenne M, Maurer JR, Boehler A, Egan JJ, Frost A, et al. (2002) Bronchiolitis obliterans syndrome 2001: an update of the diagnostic criteria. J Heart and Lung Transplant. 21: 297-310. [Crossref]

6. Reynaud-Gaubert M, Thomas P, Badier M, Cau P, Giudicelli R, et al. (2000) Early detection of airway involvement in obliterative bronchiolitis after lung transplantation: functional and bronchoalveolar lavage cell findings. Am Jf Respir Critl Care Med. 161: 1924-1929. [Crossref] 
7. Glanville AR (2010) Bronchoscopic monitoring after lung transplantation. Semin Respir Crit Care Med 31: 208-221. [Crossref]

8. Dransfield MT, Garver RI, Weill D (2004) Standardized guidelines for surveillance bronchoscopy reduce complications in lung transplant recipients. $J$ Heart and Lung Transplant. 23: 110-114. [Crossref]

9. Phillips M, Boehmer JP, Cataneo RN, Cheema T, Eisen HJ, et al. (2004) Prediction of heart transplant rejection with a breath test for markers of oxidative stress. Am J Cardiol. 94: 1593-1594. [Crossref]

10. Estenne M, Maurer JR, Boehler A, Egan JJ, Frost A, et al. (2002) Bronchiolitis obliterans syndrome 2001: an update of the diagnostic criteria. J Heart Lung Transplant 21: 297-310. [Crossref]

11. Chow S, Yates DH, Thomas PS (2008) Reproducibility of exhaled breath condensate markers. Eur Respir J 32: 1124-1126. [Crossref]

12. Liu J, Conrad DH, Chow S, Tran VH, Yates DH, et al. (2007) Collection devices influence the constituents of exhaled breath condensate. Eur Respir J30: 807-808. [Crossref]

13. Lin JL, Bonnichsen MH, Thomas PS (2011) Standardization of exhaled breath condensate: effects of different de-aeration protocols on $\mathrm{pH}$ and Hâ,,Oâ,, concentrations. J Breath Res 5: 011001. [Crossref]

14. Gebicki JM, Collins J, Baoutina A, Phair P (1996) The limitations of an iodometric aerobic assay for peroxides. Anal Biochem 240: 235-241. [Crossref]

15. Misko TP, Schilling R, Salvemini D, Moore W, Currie M (1993) A fluorometric assay for the measurement of nitrite in biological samples. Analytical Biochemistry. 214: 1116. [Crossref]

16. Kastelijn EA, Rijkers GT, Van Moorsel CH, Zanen P, Kwakkel-van Erp JM, et al. (2010) Systemic and exhaled cytokine and chemokine profiles are associated with the development of bronchiolitis obliterans syndrome. J Heart and Lung Transplant. 29: 997-1008. [Crossref]
17. Jackson AS, Sandrini A, Campbell C, Chow S, Thomas PS, et al. (2007) Comparison of biomarkers in exhaled breath condensate and bronchoalveolar lavage. Am J Respir Crit Care Med 175: 222-227. [Crossref]

18. Dressel H, Müller F, Fischer R, Römmelt H, Hohlfeld JM, et al. (2010) Independen information of nonspecific biomarkers in exhaled breath condensate. Respiration 80: 401-409. [Crossref]

19. Antus B, Barta I, Czebe K, Horvath I, Csiszer E (2010) Analysis of cytokine pattern in exhaled breath condensate of lung transplant recipients with bronchiolitis obliterans syndrome. Inflamm Research. 59: 83-86. [Crossref]

20. Meyer KC, Raghu G, Verleden GM, Corris PA, Aurora P, et al. (2014) An international ISHLT/ATS/ERS clinical practice guideline: diagnosis and management of bronchiolitis obliterans syndrome. Eur Respir J 44: 1479-1503. [Crossref]

21. Sack U, Scheibe R, Wötzel M, Hammerschmidt S, Kuhn H, et al. (2006) Multiplex analysis of cytokines in exhaled breath condensate. Cytometry A 69: 169-172. [Crossref]

22. Goldoni M, Caglieri A, Andreoli R, Poli D, Manini P, et al. (2005) Influence of condensation temperature on selected exhaled breath parameters. BMC Pulm Med 5: 10. [Crossref]

23. Franklin P, Moeller A, Hall GL, Horak F Jr, Patterson H, et al. (2006) Variability of nitric oxide metabolites in exhaled breath condensate. Respir Med 100: 123-129. [Crossref]

24. Reis GS, Augusto Vdos S, Souza ME, Baldo CF, Rodrigues AJ, et al. (2010) Exhaled breath condensate collection for nitrite dosage: a safe and low cost adaptation. Acta Cir Bras 25: 206-213. [Crossref]

Copyright: (C2017 Hettiarachchi RM. This is an open-access article distributed under the terms of the Creative Commons Attribution License, which permits unrestricted use, distribution, and reproduction in any medium, provided the original author and source are credited. 